\section{Microbiological assessment of raw goat milk collected from Sardinian herds}

\author{
Francesca Carusillo, Valentina Rosu, \\ Cipriana Fancello, Tonino Pirino, Ennio \\ Bandino, Andrea Orrù \\ Laboratorio di Microbiologia degli \\ Alimenti, Istituto Zooprofilattico della \\ Sardegna, Nuoro, Italy
}

\section{Abstract}

With Regulation EC 853/04, the European Parliament and the Council laid down general rules for food business operators regarding the hygiene of foodstuffs. In particular, the regulation established $\leq 1.500 .000 \mathrm{cfu} / \mathrm{mL}$ as the maximum-tolerated value for total bacterial count in raw goat milk. Moreover, in order to enhance the hygiene of dairy farms, the Sardinia Region has funded the $F$ measure programme which provides rewards for farmers showing improvements in herd management and animal welfare practices. This work aimed to evaluate the microbiological quality of raw goat milk samples collected during the $F$ measure enforcement. A total of 536 raw goat samples, collected from dairy farms in the Sardinian province of Nuoro and Ogliastra, were analised for total bacterial count at $30^{\circ} \mathrm{C}$. Results showed that total bacterial count was $\leq 1.500 .000 \mathrm{ufc} / \mathrm{mL}$ in 456 (85.1\%) raw milk goat samples, most of them $(80.2 \%)$ with a total bacterial contamination $<500.000 \mathrm{cfu} / \mathrm{mL}$. This study confirms the hygienic good quality of raw goat milk collected from Sardinian dairy farms.

\section{Introduzione}

La carica batterica totale (CBT) è un parametro utilizzato come indicatore generico delle condizioni igieniche di un allevamento, in particolare dell'igiene della mungitura e della conservazione del latte e fornisce in maniera indiretta un quadro delle contaminazioni da microrganismi patogeni (Bava et al., 2011). Il latte prodotto da una mammella sana è, infatti, praticamente privo di microrganismi eccetto l'accidentale contaminazione per risalita attraverso il canale del capezzolo. Nel caso di latte crudo di massa proveniente da specie diverse dal bovino, il tenore in germi a $30^{\circ} \mathrm{C}$ deve risultare $\leq 1.500 .000 \mathrm{ufc} / \mathrm{mL}$, limite discriminante di conformità indicato dal Regolamento CE 853/04 (Commissione Europea, 2004b), da definirsi mediante media geometrica mobile su campioni prelevati a distanza di 15 giorni in un periodo di due mesi. Una carica microbica elevata nel latte di massa può essere conseguenza di una mancata applicazione di buone prassi di conduzione zootecnica (Chambers, 2005) come ad esempio, scarse condizioni igieniche della stalla, cattive pratiche di mungitura, scarsa pulizia delle attrezzature, non adeguata refrigerazione del latte (malfunzionamento del refrigeratore), utilizzo di acqua non potabile che non rispetta i limiti microbiologici imposti dalla normativa vigente. I Regolamenti comunitari 852/04 e 853/04 (Commissione Europea, 2004a, 2004b) riportano i requisiti che l'operatore del settore alimentare (OSA) è tenuto a rispettare per immettere sul mercato un prodotto sicuro per il consumatore. Il latte crudo destinato agli stabilimenti di trattamento e trasformazione deve possedere i seguenti requisiti: i) provenire esclusivamente da aziende registrate; ii) rispettare i limiti di tenore in germi e, per il latte di vacca, cellule somatiche (Regolamento CE 853/04; Commissione Europea, 2004b); iii) essere esente da agenti patogeni trasmissibili all'uomo attraverso il consumo di latte; iv) non contenere contaminanti ambientali pericolosi, né residui di trattamenti farmacologici in quantità superiori ai limiti di legge (Regolamento UE N. 37/2010; Commissione Europea, 2010). I Regolamenti comunitari 852/04 e 853/04 obbligano quindi l'OSA a prestare attenzione alle caratteristiche igienicosanitarie legate in particolar modo alla raccolta del latte e alle condizioni di allevamento. A tal proposito la politica agricola dell'Unione Europea ha individuato nella condizionalità lo strumento che sostiene, anche economicamente, il settore primario nel processo di miglioramento qualitativo delle produzioni.

Considerato che esiste una stretta relazione tra stato di benessere degli animali allevati e sicurezza alimentare, la Regione Sardegna ha rafforzato la politica comunitaria della condizionalità nel proprio territorio con apposito decreto n. 3177 del 18 dicembre 2008 inserendo nel Piano di Sviluppo Rurale, la misura $F$, che prevede la determinazione di indicatori dello stato di benessere animale (Regione Sardegna, 2008).

Per supportare ulteriormente la misura $F$ della politica regionale, l'Istituto Zooprofilattico Sperimentale della Sardegna, in aggiunta alle proprie competenze istituzionali previste dal Pacchetto Igiene ovvero il sistema che individua i principi e i requisiti generali in materia di sicurezza alimentare stabiliti dal Regolamento CE 178/2002 (Commissione Europea, 2002) e rafforzato da ulteriori provvedimenti normativi rappresentati dai Regolamenti comunitari CE 852/2004, 853/2004, 183/2005 (Commissione Europea, 2004a, 2004b, 2005), effettua su campioni di latte di massa caprino crudo la determinazione della CBT a $30^{\circ} \mathrm{C}$.
Correspondence: Andrea Orrù, Laboratorio di Microbiologia degli Alimenti, Istituto Zooprofilattico della Sardegna, via F.lli Kennedy 2,08100 Nuoro, Italy.

Tel. +39.0784.233802 - Fax: +39.0784.399006.

E-mail: andrea.orru@izs-sardegna.it

Key words: Hygiene of foodstuffs, Raw goat milk, Total bacterial count.

Conflict of interests: the authors declare no potential conflict of interests.

Contributions: FC, research method and samples collection; VR, preparation of the manuscript and data analysis; CF and TP, determination of milk total bacterial count; $\mathrm{EB}$, critical revision of the manuscript; A0, research method, data interpretation, critical revision of the manuscript. All Authors read and approved the final version of the manuscript.

Received for publication: 10 May 2013.

Revision received: 21 November 2013.

Accepted for publication: 20 December 2013.

This work is licensed under a Creative Commons Attribution 3.0 License (by-nc 3.0).

(C) Copyright F. Carusillo et al., 2014

Licensee PAGEPress, Italy

Italian Journal of Food Safety 2014; 3:1638

doi:10.4081/ijfs.2014.1638

Nel presente lavoro sono riportati i dati relativi alla determinazione della CBT a $30^{\circ} \mathrm{C}$ effettuata su campioni di latte provenienti da allevamenti delle province di Nuoro ed Ogliastra, nel quinquennio 2008-2012, prelevati nell'ambito della misura $F$. L'obiettivo è stato quello di valutare le caratteristiche igienico-sanitarie dei campioni di latte e la qualità dal punto di vista microbiologico.

\section{Materiali e Metodi}

Nel quinquennio 2008-2012 sono stati analizzati presso il laboratorio di Microbiologia degli Alimenti del Dipartimento Territoriale di Nuoro, Istituto Zooprofilattico Sperimentale della Sardegna, un totale di 536 campioni di latte crudo di massa caprino provenienti da allevamenti delle province di Nuoro ed Ogliastra, in Sardegna. Il monitoraggio è stato effettuato nell'ambito della campagna misura $F$ e i campionamenti sono stati effettuati, con cadenza mensile, da gennaio ad agosto ed eseguiti direttamente dagli allevatori convenzionati con l'Istituto Zooprofilattico Sperimentale della Sardegna, secondo metodologia standardizzata.

I campioni di latte refrigerato venivano conferiti al laboratorio in contenitori asettici entro 
24 ore dal prelievo. Al momento della consegna del campione è stata verificata la temperatura ed i campioni con valori compresi tra 2 e $8^{\circ} \mathrm{C}$, con un rialzo termico di $2^{\circ} \mathrm{C}$ tollerabile per periodi di breve durata, sono stati considerati idonei e processati.

Il Laboratorio di Microbiologia degli Alimenti del Dipartimento Territoriale di Nuoro opera in conformità alla norma ISO 17025:2005 (ISO, 2005). Il metodo analitico accreditato ed utilizzato per la determinazione della $\mathrm{CBT}$ a $30^{\circ} \mathrm{C}$ è quello previsto nel Decreto Ministeriale del 26 Marzo 1992-Allegato 2 del Ministero della Sanità, in attuazione della Decisione n.91/180/CEE (Repubblica Italiana, 1992). Il valore discriminante adottato per definire un campione idoneo è quello riportato nel Regolamento CE 853/04 (Commissione Europea, 2004a), che, per il latte crudo ovino e caprino destinato alla trasformazione previo trattamento termico, risulta $\leq 1.500 .000$ ufc/mL. Per il latte di massa caprino destinato alla produzione di formaggi a base di latte crudo tale valore è $\leq 500.000 \mathrm{ufc} / \mathrm{mL}$.

\section{Risultati}

L'analisi dei dati ha evidenziato che l' $85,1 \%$ dei campioni analizzati presentava una CBT a $30^{\circ} \mathrm{C} \leq 1.500 .000 \mathrm{ufc} / \mathrm{mL}$, valore massimo ammesso per il latte da sottoporre a trattamento termico (Tabella 1 e Figura 1). Nel periodo 2008-2012 la percentuale di campioni idonei è rimasta costante (circa 85\%) ad eccezione dell'anno 2008 quando il numero di campioni idonei è risultato leggermente superiore alla media (Tabella 1 e Figura 1), ma con una campionatura ridotta (n. 36 campioni totali) rispetto agli anni successivi. Infatti, come evidenziato nella Tabella 1 e nella Figura 1, il numero dei campioni sottoposti a controllo è aumentato di anno in anno e ciò può essere conseguenza di una maggiore presa di coscienza da parte degli allevatori del loro ruolo di OSA. All'interno del gruppo di campioni con CBT a $30^{\circ} \mathrm{C} \leq 1.500 .000 \mathrm{ufc} / \mathrm{mL}$, una percentuale pari al $80,2 \%$ presentava una CBT $<500.000$ ufc $/ \mathrm{mL}$.

\section{Conclusioni}

L'analisi dei dati ottenuti nel quinquennio 2008-2012 ha evidenziato che l' $85,1 \%$ dei campioni di latte di massa testati presentava una $\mathrm{CBT}$ a $30^{\circ} \mathrm{C}$ inferiore al valore massimo discriminante adottato per il latte caprino destinato alla trasformazione previo trattamento termico. All'interno di questo gruppo, il 80,2\% dei campioni ha fatto registrare valori addirittura inferiori al limite adottato per il latte di massa

Tabella 1. Distribuzione dei valori della carica batterica totale nel latte di capra nel quinquennio 2008-2012.

\begin{tabular}{|c|c|c|c|c|}
\hline Anno & Campioni analizzati (n) & $>1.500 .000$ & $\begin{array}{c}\text { CBT }(\mathrm{cfu} / \mathrm{mL}) \\
\mathrm{da} \leq 1.500 .000 \mathrm{a}>500.000\end{array}$ & $<500.000$ \\
\hline 2008 & 36 & $3(8,3)$ & $3(8,3)$ & $30(83,3)$ \\
\hline 2009 & 97 & $18(18,6)$ & 0 & $79(81,4)$ \\
\hline 2010 & 115 & $12(10,4)$ & $19(16,5)$ & $84(73,0)$ \\
\hline 2011 & 119 & $14(11,8)$ & $4(3,4)$ & $101(84,9)$ \\
\hline 2012 & 169 & $25(14,8)$ & $8(4,7)$ & $136(80,5)$ \\
\hline Totale & 536 & $80(14,9)$ & $26(4,9)$ & $430(80,2)$ \\
\hline
\end{tabular}

CBT, carica batterica totale. I valori tra parentesi indicano la percentuale dei campioni.

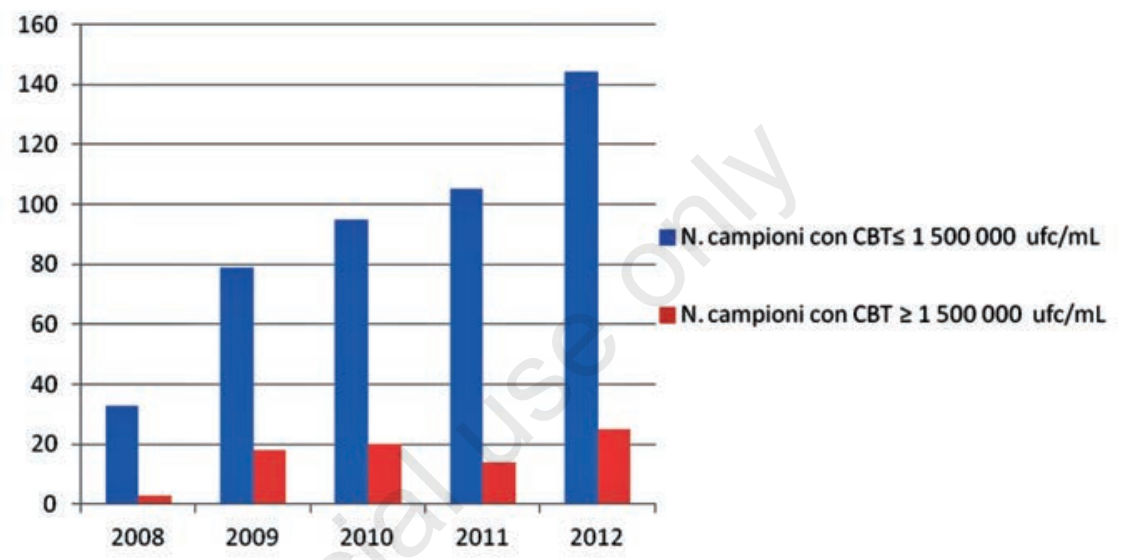

Figura 1. Numero di campioni con carica batterica totale inferiore e superiore a $\leq 1.500 .000 \mathrm{ufc} / \mathrm{mL}$ nel periodo $2008-2012$.

caprino destinato alla preparazione di formaggi a base di latte crudo. In sintesi, il latte degli allevamenti caprini esaminato nel quinquennio 2008-2012 presenta una condizione igienico-sanitaria accettabile per la trasformazione casearia. Solamente il $14,9 \%$ dei campioni ha superato il valore massimo discriminante, indicando che alcune aziende sarde hanno ancora bisogno di adottare delle procedure e/o degli strumenti mirati a migliorare le condizione igieniche d'allevamento e di raccolta del latte, in modo da immettere sul mercato un prodotto con migliori caratteristiche igienicosanitarie. La mancata 0 inefficiente applicazione di buone prassi di conduzione zootecnica (per esempio, un controllo degli agenti mastidogeni non adeguato, cattive pratiche di mungitura, scarsa pulizia delle attrezzature, inadeguata refrigerazione) potrebbe essere la causa dell'elevata carica microbica rilevata. È auspicabile un maggior processo di maturazione imprenditoriale, che consenta di individuare nel miglioramento del benessere animale, evidenziato dalla determinazione dei singoli parametri, e nelle buone pratiche di conduzione zootecnica, un incremento della produttivi- tà e redditività aziendale, ulteriormente supportata da una politica di remunerazione del latte in base alle caratteristiche qualitative.

\section{Bibliografia}

Bava L, Zucali M, Sandrucci A, Brasca M, Vanoni L, Zanini L, Tamburini A, 2011. Effect of cleaning procedure and hygienic condition of milking equipment on bacterial count of bulk tank milk. J Dairy Res 4:1-9.

Chambers JV, 2005. Microbiology of raw milk. In: Robinson RK, ed. Dairy microbiology handbook: the microbiology of milk and milk products. John Wiley \& Sons, Hoboken, NJ, USA, pp 39-90.

Commissione Europea, 2002. Regulation of the European Parliament and of the Council of 28 January 2002 laying down the general principles and requirements of food law, establishing the European Food Safety Authority and laying down procedures in matters of food safety, 178/2002/CE. In: 
Gazzetta Ufficiale, L 31/1, 01/02/2002.

Commissione Europea, 2004a. Regulation of the European Parliament and of the Council of 29 April 2004 on the hygiene of foodstuffs, 852/2004/CE. In: Gazzetta Ufficiale, L 139, 30/04/2004.

Commissione Europea, 2004b. Regulation of the European Parliament and of the Council of 29 April 2004 laying down specific hygiene rules for on the hygiene of foodstuffs, 853/2004/CE. In: Gazzetta Ufficiale, L 139/55, 30/04/2004.

Commissione Europea, 2005. Regulation of the European Parliament and of the Council of 12 January 2005 laying down requirements for feed hygiene, 183/2005/CE. In:
Gazzetta Ufficiale, L 35/1, 08/02/2005.

Commissione Europea, 2010. Regulation of the European Commission of 22 December 2009 on pharmacologically active substances and their classification regarding maximum residue limits in foodstuffs of animal origin, 37/2010/CE. In: Gazzetta Ufficiale, L 15/3, 20/1/2010.

ISO, 2005. General requirements for the competence of testing and calibration laboratories ISO Norm 17025:2005. Organizzazione per la Standardizzazione Internazionale, Ginevra, Svizzera.

Regione Sardegna, 2008. Decreto $n$. 3177/DecA/107 del 18 dicembre 2008. Disposizioni regionali per l'attuazione della condizionalità ai sensi del Reg. (CE) n. $1782 / 2003$ applicabile dal $1^{\circ}$ gennaio 2009. Recepimento del decreto ministeriale n. 12541 del 21 dicembre 2006 e successive modifiche e integrazioni. Disponibile al sito: http://www.regione. sardegna.it/documenti/1_5_20081 224133744.pdf

Repubblica Italiana, 1992. Decreto Ministeriale del 26 Marzo 1992. Attuazione della decisione n. 91/180/CEE concernente la fissazione di metodi di analisi e prova relativi al latte crudo e al latte trattato termicamente. In: Gazzetta Ufficiale, n. 90, Suppl. Ordinario n. 67, 16/04/1992. 